\title{
Lesion detection at seven Tesla in multiple sclerosis using magnetisation prepared 3D-FLAIR and 3D-DIR
}

\author{
Wolter L. de Graaf • Jaco J. M. Zwanenburg • Fredy Visser • Mike P. Wattjes • \\ Petra J. W. Pouwels • Jeroen J. G. Geurts • Chris H. Polman • Frederik Barkhof • \\ Peter R. Luijten • Jonas A. Castelijns
}

Received: 15 March 2011 /Revised: 27 June 2011 / Accepted: 6 July 2011 /Published online: 27 August 2011

(C) The Author(s) 2011. This article is published with open access at Springerlink.com

\begin{abstract}
Objectives To examine the feasibility and value of $7 \mathrm{~T} 3 \mathrm{D}$ T2-weighted Fluid Attenuated Inversion Recovery (FLAIR) and Double Inversion Recovery (DIR) MR sequences for lesion detection in multiple sclerosis (MS).

Methods High-resolution 3D-FLAIR and 3D-DIR MR sequences at $7 \mathrm{~T}$ were obtained using magnetisation preparation (MP), and compared with 2D-T2-weighted and 3D-T1-weighted sequences in $10 \mathrm{MS}$ patients and five healthy controls. We determined contrast ratios and counted lesions according to anatomical location.

Results MR imaging at $7 \mathrm{~T}$ was safe and allowed multicontrast imaging within clinically acceptable imaging times. Lesion to white matter (WM) and grey matter (GM) contrast ratios were higher in 3D-MP-FLAIR and 3D-MP-DIR compared with 2D-T2 and 3D-T1. Cortical (mixed+intra-cortical) and total lesion counts were 97/592
\end{abstract}

W. L. de Graaf · M. P. Wattjes · F. Barkhof · J. A. Castelijns Department of Radiology, VU

University Medical Center Amsterdam,

De Boelelaan 1117, NL-1081 HV, Amsterdam, the Netherlands

J. J. M. Zwanenburg • F. Visser • P. R. Luijten

Image Sciences Institute, University Medical Center Utrecht, P.O. Box 85500, NL-3508 GA,

Utrecht, the Netherlands

J. J. M. Zwanenburg $\cdot$ F. Visser $\cdot$ P. R. Luijten

Department of Radiology, University Medical Center Utrecht, P.O. Box 85500, NL-3508 GA,

Utrecht, the Netherlands

F. Visser

Philips Healthcare,

Best, the Netherlands on 3D-MP-FLAIR and 100/558 on 3D-MP-DIR compared with $84 / 384$ on $2 \mathrm{D}-\mathrm{T} 2$ and $42 / 442$ on 3D-T1. More juxtacortical lesions were seen with 3D-MP-FLAIR (205) and 3D-MP-DIR (133) than with 2D-T2 (125) and 3D-T1 (70). Finally, higher numbers of lesions were found for deep WM lesions: 176 for 3D-MP-FLAIR and 196 for 3D-MP-DIR vs. 155 for $2 \mathrm{D}-\mathrm{T} 2$ and 131 for $3 \mathrm{D}-\mathrm{T} 1$.

Conclusions Near isotropic 3D-MP-FLAIR and 3D-MPDIR allows high quality T2-weighted MR imaging in MS at $7 \mathrm{~T}$, improving (cortical) lesion detection.

Key Points

- Magnetization prepared 3D-FLAIR and 3D-DIR $7 T$ MRI provide high quality isotropic images.

- MS lesions are well demonstrated by 3D-MP-FLAIR and $3 D-M P-D I R$ at $7 T$ MRI.

- 3D-MP-FLAIR and 3D-MP-DIR at 7 T MRI show many more Virchov-Robin spaces.

P. J. W. Pouwels

Department of Physics and Medical Technology,

VU University Medical Center Amsterdam,

De Boelelaan 1117, NL-1081 HV, Amsterdam, the Netherlands

\section{J. J. G. Geurts}

Department of Anatomy and Neurosciences,

VU University Medical Center Amsterdam,

De Boelelaan 1117, NL-1081 HV, Amsterdam, the Netherlands

\section{H. Polman}

Department of Neurology,

VU University Medical Center Amsterdam,

De Boelelaan 1117, NL-1081 HV, Amsterdam, the Netherlands

W. L. de Graaf $(\bowtie)$

Department of Radiology,

VU University Medical Center Amsterdam,

P.O. Box 7057, NL-1007 MB, Amsterdam, The Netherlands

e-mail: W.deGraaf@vumc.nl 
Keywords Magnetic Resonance Imaging/methods . Imaging · Three-Dimensional · Multiple Sclerosis/ diagnosis $\cdot$ Multiple Sclerosis/pathology $\cdot$ Cerebral Cortex/ pathology

\section{Introduction}

Multiple Sclerosis (MS) is a chronic immune-mediated inflammatory disease of the central nervous system (CNS) that causes demyelination of axonal myelin sheaths and neurodegeneration. MR imaging is a valuable diagnostic tool that is able to non-invasively show demyelinating lesions in the brain and spinal cord. Pulse sequences like fluid attenuated inversion recovery (FLAIR) and double inversion recovery (DIR) suppress the signal of cerebrospinal fluid (CSF), while DIR also attenuates the signal of white matter (WM), and have proved to be more sensitive for brain lesions in WM and grey matter (GM) than proton density (PD) or T2-weighted (T2w) and T1-weighted (T1w) MR sequences $[1-5]$ at $1.5 \mathrm{~T}$ and $3.0 \mathrm{~T}$.

While the emphasis in MS has long been directed mainly at WM, there is an increasing interest for GM abnormalities: In vivo imaging of cortical lesions has become feasible and relations between GM damage and clinical manifestation have been shown [6-8]. Detection of WM and especially GM lesions requires a high spatial resolution and a good signal-to-noise ratio (SNR) as most of the GM lesions are not of the inflammatory type. This all should be done within a clinically acceptable imaging time $[4,9,10]$. Unfortunately, the additional 180-pulses in FLAIR and DIR sequences also lower the signal intensity of the nonsuppressed tissues. Therefore, MR imaging at high field strength $(7 \mathrm{~T})$ might be favourable as it provides an intrinsically higher SNR that can be used for higher spatial resolution and/or for faster imaging by using higher parallel imaging rates [11]. MR examinations at $3 \mathrm{~T}$ detected more lesions than at $1.5 \mathrm{~T}$, although the added value for the diagnosis MS remains uncertain [12]. Also treatment monitoring may benefit from possible increased sensitivity as new lesions may be seen earlier. Finally, detection of (small) GM lesions may help to increase correlation with the clinical status of the patient. To date, most clinical $7 \mathrm{~T}$ studies with MS patients have been confined to the T1w, PDT2 $w$, and T2*w protocols [13-16]. So far, 3D-FLAIR and 3D-DIR, both showing increased cortical lesion detection at standard MR field strength (1.5 $\mathrm{T}$ and $3 \mathrm{~T})$, could not easily be implemented at $7 \mathrm{~T}$. This is largely because acquisition of 3D-FLAIR and 3D-DIR is challenging at high field strength, owing to a number of issues like their sensitivity to heterogeneity in the amplitude of the transmit RF field $\left(B_{1}\right)$, the high specific absorption rate (SAR), long T1 relaxation, and lengthy repetition times. Consequently, this leads to long acquisition times in the standard implementation of these sequences [17]. An initial experience of DIR imaging in healthy volunteers at $7 \mathrm{~T}$ and $3 \mathrm{~T}$ demonstrated some advantage of 2D-DIR at high field strength, but showed unsatisfactory results for 3D-DIR [18]. 2D-FLAIR was also tested but used a slice thickness of $4 \mathrm{~mm}$ [16]. It needed $16 \mathrm{~min}$ of scan time to cover the brain as for each slice a separate adiabatic inversion pulse needs to be transmitted while using long repetition times to compensate for long $\mathrm{T} 1$ relaxation times and to cover SAR limitations. Recently, a 3D magnetisation prepared FLAIR (3D-MP-FLAIR) sequence was described and optimized for use at $7 \mathrm{~T}$ [19]. In a clinically acceptable acquisition time

Table 1 Sequence parameters used in the imaging protocol

\begin{tabular}{|c|c|c|c|c|}
\hline Contrast & 3D-MP-FLAIR & 3D-MP-DIR & 3D-T1 & 2D-PDT2 \\
\hline Sequence & TSE & TSE & TFE & TSE \\
\hline TR [ms] & 8,000 & 8,000 & 7.0 & 4,969 \\
\hline $\mathrm{TE}(1 / 2)[\mathrm{ms}]$ & 303 & 294 & 2.9 & $21 / 80$ \\
\hline TI $(1 / 2)[\mathrm{ms}]$ & 2,325 & $3,150 / 550$ & 1,129 & - \\
\hline Flip Angle [deg] & 100 & 100 & 8 & 90 \\
\hline Echo Train Length $[-]$ & 125 & 128 & 312 & 8 \\
\hline $\mathrm{FOV}\left[\mathrm{mm}^{3}\right]$ & $250 \times 250 \times 190$ & $250 \times 250 \times 190$ & $250 \times 200 \times 190$ & $230 \times 200 \times 120$ \\
\hline Matrix [-] & $312 \times 304 \times 238$ & $252 \times 250 \times 238$ & $248 \times 312 \times 238$ & $328 \times 227 \times 60$ \\
\hline SENSE $[-]$ & $2.5 \times 2.5(\mathrm{APxRL}) *$ & $2.0 \times 3.0(\mathrm{APxRL})^{*}$ & $2.0(\mathrm{RL})$ & $2.1(\mathrm{RL})$ \\
\hline Acq. Res $\left[\mathrm{mm}^{3}\right]$ & $0.8 \times 0.8 \times 0.8$ & $1.0 \times 1.0 \times 0.8$ & $0.8 \times 0.8 \times 0.8$ & $0.7 \times 1.0 \times 2.0$ \\
\hline Recon. Res. $\left[\mathrm{mm}^{3}\right]$ & $0.45 \times 0.45 \times 0.4$ & $0.5 \times 0.5 \times 0.4$ & $0.5 \times 0.5 \times 0.4$ & $0.45 \times 0.45 \times 2.0$ \\
\hline Acq. Time [min:sec] & $13: 06$ & 11:04 & $9: 43$ & $8: 07$ \\
\hline
\end{tabular}

*SENSE applied in 2 dimensions (AP: Anterior-Posterior, $L R$ : Left-Right)

TR: Repetition Time, TE: Echo Time, TI: Inversion Time, FOV: Field of View, SENSE: Sensitivity Encoding, Acq. Res: Acquired Resolution, Recon. Res: Reconstructed Resolution, Acq. Time: Acquisition duration 
this 3D-MP-FLAIR sequence gives the desired contrast in healthy subjects.

The aim of this explorative study was to examine the feasibility and value of new 3D T2-weighted MP-FLAIR and MP-DIR sequences at $7 \mathrm{~T}$ MR imaging for lesion detection in MS patients. The potential of these sequences to detect lesions in WM and GM was explored and compared with conventional 2D-PDT2 and 3D-T1-weighted sequences at $7 \mathrm{~T}$ in the same MS patients.

\section{Material and methods}

Patients and healthy controls

Ethical approval for the study was received from the institutional ethical review board of the hospital and all subjects gave written informed consent before the examination. Ten MS patients (most with the primary progressive disease type) from our Neurology outpatient clinic and five healthy controls were consecutively included in this prospective study. Inclusion criteria were clinically definite MS according to the revised 'McDonald criteria' of 2005 [20] and age between 18 and 60 years. Exclusion criteria were other neurological diseases (patients as well as healthy volunteers) and recent relapses ( $<3$ months). Local high field MR safety regulations were applied that on top of the standard field regulations exclude all subjects with metal objects in or on the body and also exclude subjects with suspicion of presence of metal objects that were possibly applied during surgical interventions. The use of disease modifying treatment was not an exclusion criterion. No contrast agents were used in this study. Patients ( 3 men, 7 women) had a mean age of 48 years (range 43-53) and an Expanded Disability Status Scale (EDSS) median of 4.5 (range 3.5-7.5). Healthy control subjects (3 men, 2 women) had a mean age of 44 years (range 33-52). After imaging each subject was asked about any discomfort other than that experienced at lower field strength.

\section{MRI acquisition}

All subjects were examined with a $7 \mathrm{~T}$ whole body MR imaging System (Philips Achieva, Philips Healthcare, Cleveland, OH, USA), with a maximum B1 of $20 \mu \mathrm{T}$. Radiofrequency (RF) was transmitted through a volume transmit coil and received using a 16-channel phased array receive head coil (Nova Medical, Inc. Wilmington, MA, USA). Subjects were inserted slowly into the bore $(<10 \mathrm{~cm} / \mathrm{s})$ and informed that mild dizziness might occur during table movement. In this study we used 2D- PDT2w, 3D-T1w, 3DMP-FLAIR and 3D-MP-DIR sequences. FLAIR and DIR sequences used a new magnetisation preparation [19, 21],

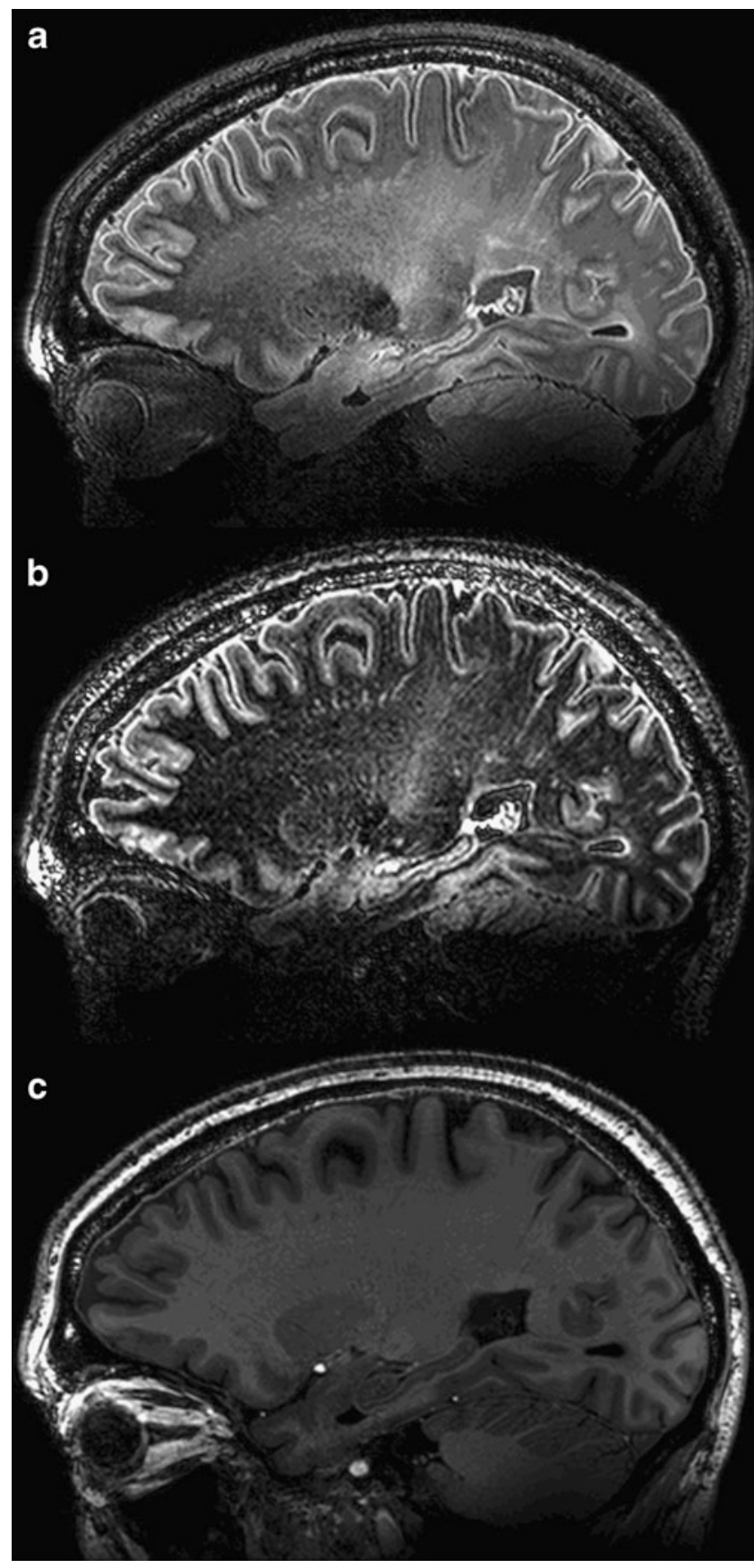

Fig. 1 Sagittal $7 \mathrm{~T}$ brain images of a healthy male control (aged 50 years), a 3D-MP-FLAIR (TR: $8,000 \mathrm{~ms}$, TE: $303 \mathrm{~ms}$, TI: $2,325 \mathrm{~ms}$ ), b 3D-MP-DIR (TR: 8,000 ms, TE: $294 \mathrm{~ms}$, TI: 1/2: 3,150/550 ms), c 3D-T1 (TR: $7.0 \mathrm{~ms}$, TE: $2.9 \mathrm{~ms}$, TI: $1,129 \mathrm{~ms}$ ). Although fluid attenuated inversion recovery (FLAIR) and double inversion recovery (DIR) both aim to suppress CSF signal to detect lesions close to sulci and ventricles, CSF attenuation is not homogeneous throughout the brain (a and $\mathbf{b}$ ). To a lesser extent, the variable signal intensity of CSF can also be recognised on 3D-T1 c. Especially on 3D-MP-FLAIR and 3D-MP-DIR, peri-vascular spaces (PVS) appeared hyper-intense compared with white matter (WM), while on T1 they were hypo-intense compared with the surrounding tissue

which consisted of a non selective 90 lock pulse, four adiabatic refocusing pulses followed by a $-90^{\circ}$ back pulse 
Table 2 Mean Contrast Ratios in healthy controls and MS Patients
Abs(SI1-SI2/SI2), GM: Grey Matter, WM: White Matter

\begin{tabular}{llllr}
\hline \multicolumn{5}{l}{ Contrast Ratio [value (sd)] } \\
\cline { 2 - 5 } & GM/WM (Ctrl) & GM/WM (Pat) & Lesion/WM & Lesion/GM \\
\hline 2D-PD & $0.23(0.2)$ & $0.26(0.2)$ & $0.10(1.7)$ & $0.12(0.7)$ \\
2D-T2 & $0.32(0.1)$ & $0.28(0.2)$ & $0.54(3.0)$ & $1.68(1.8)$ \\
3D-T1 & $0.28(0.1)$ & $0.38(0.2)$ & $0.49(1.1)$ & $0.31(1.0)$ \\
3D-MP-FLAIR & $0.40(0.1)$ & $0.35(0.3)$ & $0.86(1.6)$ & $0.91(2.2)$ \\
3D-MP-DIR & $0.93(0.9)$ & $0.87(0.6)$ & $2.92(2.4)$ & $1.40(7.0)$ \\
\hline
\end{tabular}

with a total duration of $100 \mathrm{~ms}$. This resulted in saturation of tissues with a short T2 relaxation time (WM and GM) before the inversion recovery pulse, while CSF and similar fluids with a long $\mathrm{T} 1$ relaxation time were hardly affected. Therefore, CSF experienced inversion recovery, while GM and WM only experienced saturation recovery. During data sampling the T1 weighting effect in WM and GM was therefore strongly reduced [19]. Table 1 shows an overview of MR parameters used for this protocol. Total examination time was about $42 \mathrm{~min}$.

\section{Analysis method}

Sagittal 3D images were reconstructed in the axial plane, corresponding to the 2D-PDT2 images. The appearance of normal and non-normal tissue (contrast of GM and WM, focal lesions, diffuse lesions, diffusely appearing WM and GM and suppression of CSF) was evaluated. A 6-level score was used to address motion artefacts and homogeneity of the images: Motion Artefacts: 0: none, 1: almost not noticeable, 2: incidental motion, 3: some artefacts, 4: multiple artefacts, needs careful analysis, 5: high numbers of artefacts, precluding valid analysis. Homogeneity: 0: good homogeneity, 1: almost homogeneous images, 2: minor heterogeneity close to skull base and in temporal lobes, 3: heterogeneous signal at multiple locations, 4: high number of heterogeneous areas, 5: highly heterogeneous images, no valid analysis possible.

In addition, all sequences were analysed with regard to contrast ratios (CR) of WM lesions, GM lesions and GM to WM. CR was defined as the absolute value of $\left(\mathrm{SI}_{1}-\mathrm{SI}_{2}\right) / \mathrm{SI}_{2}$ with $\mathrm{SI}_{1}$ and $\mathrm{SI}_{2}$ the signal intensity of the first and second mentioned term in the description (i.e. GM/WM, lesion/ $\mathrm{WM}$, and lesion/GM). On the 3D-T1 images regions of interest (ROIs) of at least $10 \mathrm{~mm}^{2}$ were drawn for GM, WM, and in MS patients for lesions. The outer rim of the cortex was not included to prevent overestimation of GM signal due to the hyperintense rim found on 3D-MP-FLAIR and 3D-MP-DIR which did not contribute to contrast between GM and WM. The ROI areas were copied to the other registered 3D sequences for CR calculation by use of MIPAV software (CIT, NIH, Bethesda, MD, USA). For 2D images this was done manually as automatic registration did not always succeed. As the appearance of normal anatomy and lesions was fundamentally different compared to conventional sequences at lower field, lesions were counted in consensus by two observers ( 4 years and 8 years of experience). The order of sequences and subjects was randomised. Lesions were counted and categorised according to their anatomical locations: periventricular (PV) lesions in contact with the ventricles or within a distance of $3 \mathrm{~mm}$; deep white matter (DWM) lesions further than

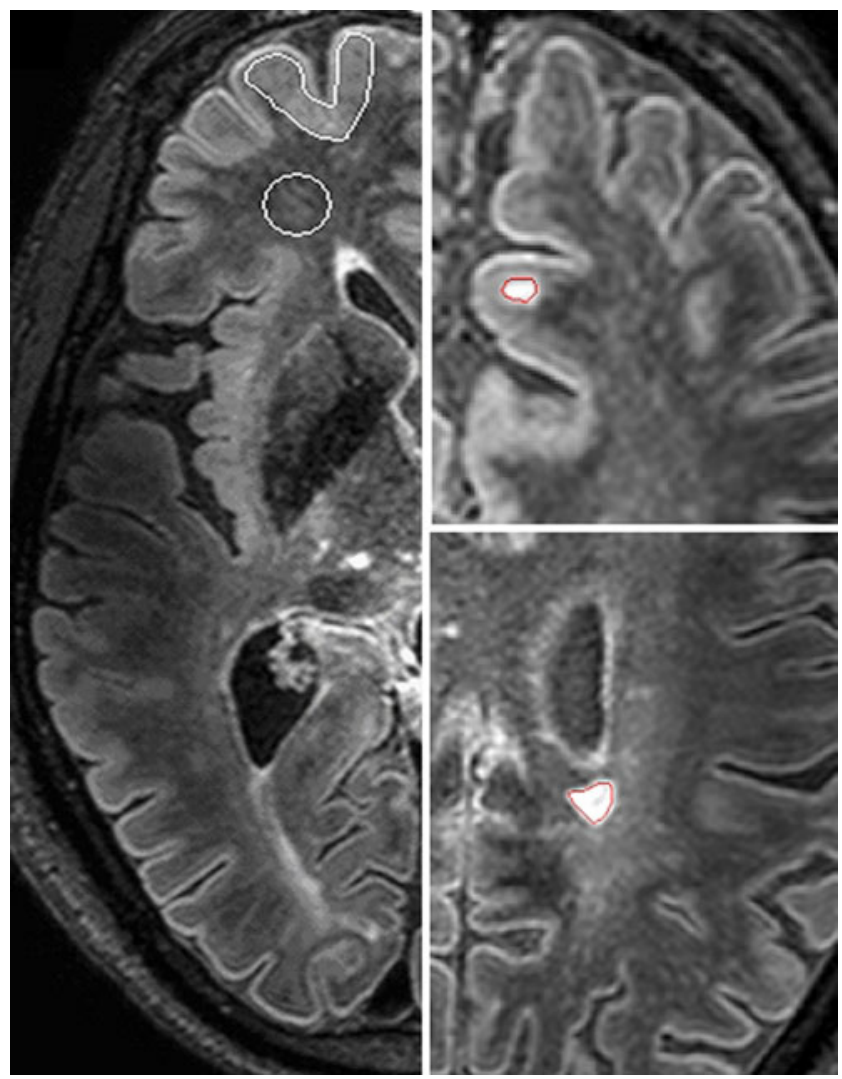

Fig. 2 Regions of interest for contrast ratio measurements on an axial reformatted 3D-MP-FLAIR image of a male primary progressive (PP) MS patient (50 years of age): LEFT: normal-appearing grey matter (NAGM) and normal-appearing white matter (NAWM). Note the fact that the hyper-intense rim of the cortex was excluded from the ROI. RIGHT: Lesions were marked within one voxel from the border to prevent partial volume effects 


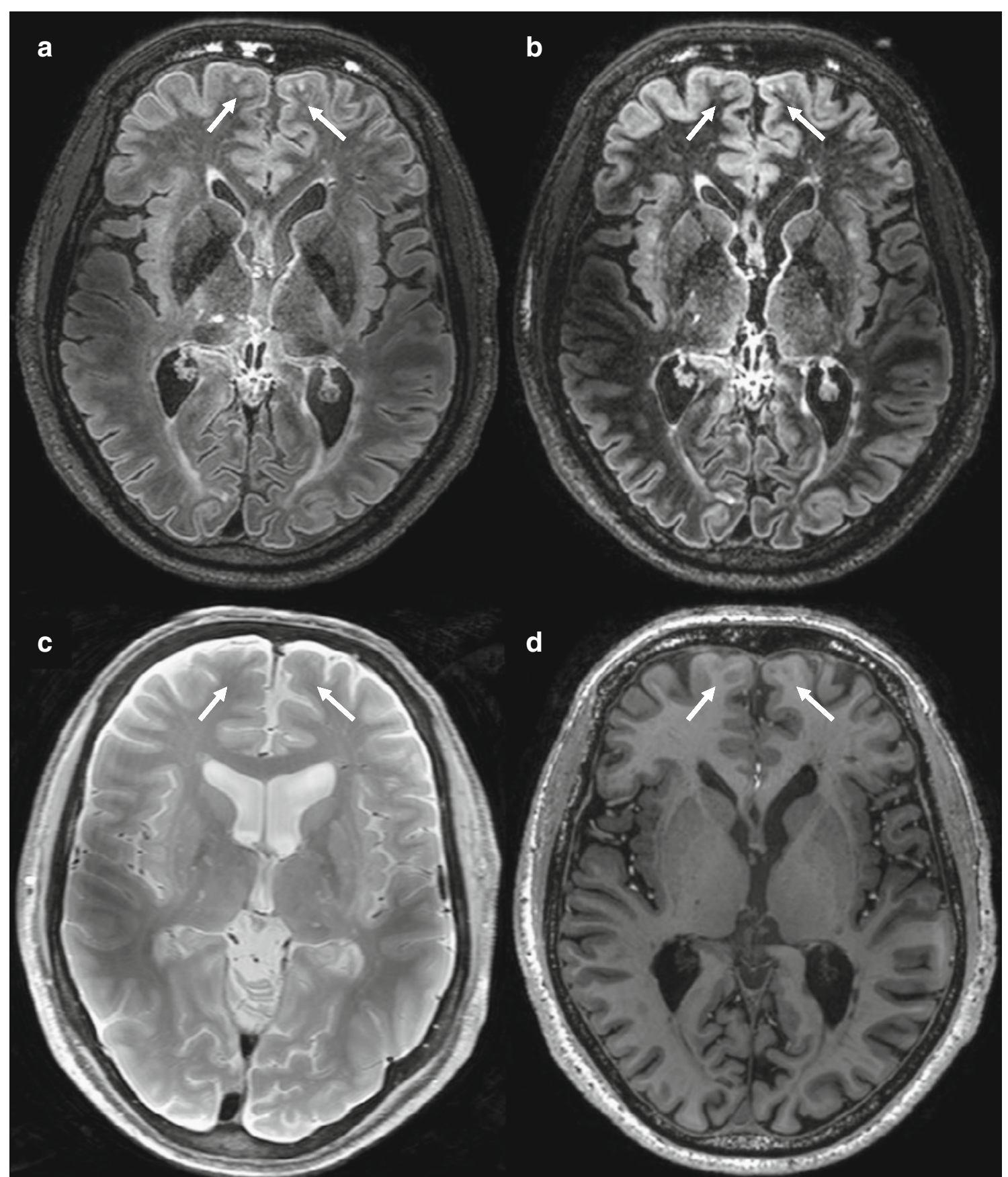

Fig. 3 Axial images (a, b and d reformatted) of a 53-year-old male PP MS patient. A number of lesions (left arrow: mixed, right arrow: juxta-cortical) can be observed in the prefrontal area of the brain on 3D-MP-FLAIR and 3D-MP-DIR images, but less easily on 3D-T1 or
PDT2 images. a 3D-MP-FLAIR (TR: 8,000 ms, TE: $303 \mathrm{~ms}$, TI: 2,325 ms), b 3D-MP-DIR (TR: 8,000 ms, TE: $294 \mathrm{~ms}$, TI 1/2: 3,150/ $550 \mathrm{~ms}$ ), c 2D-PD (TR: 4,969 ms, TE: $21 \mathrm{~ms}$ ), d 3D-T1 (TR: $7.0 \mathrm{~ms}$, TE: $2.9 \mathrm{~ms}$, TI: $1,129 \mathrm{~ms})$

infratentorial lesions were infrequently found but were not used for this comparison, as the field of view did not extend to those areas for all subjects. On 3D-T1 images, all lesions were defined as hypo-intensities compared with surrounding WM and GM, whereas on the other sequences they appeared as hyper-intensities. Finally, the number of lesions detected by the 3D methods FLAIR, DIR and T1 were compared with 2D- 
Fig. 4 Appearance of a lesion in the left temporal lobe on four image types (a, $\mathbf{b}$ and d reformatted). Top left: 3D-MPFLAIR, top right: 3D-MP-DIR, bottom left: 2D-T2, bottom right: 3D-T1. Note that despite inhomogeneity of the signal, detection of lesions is not compromised

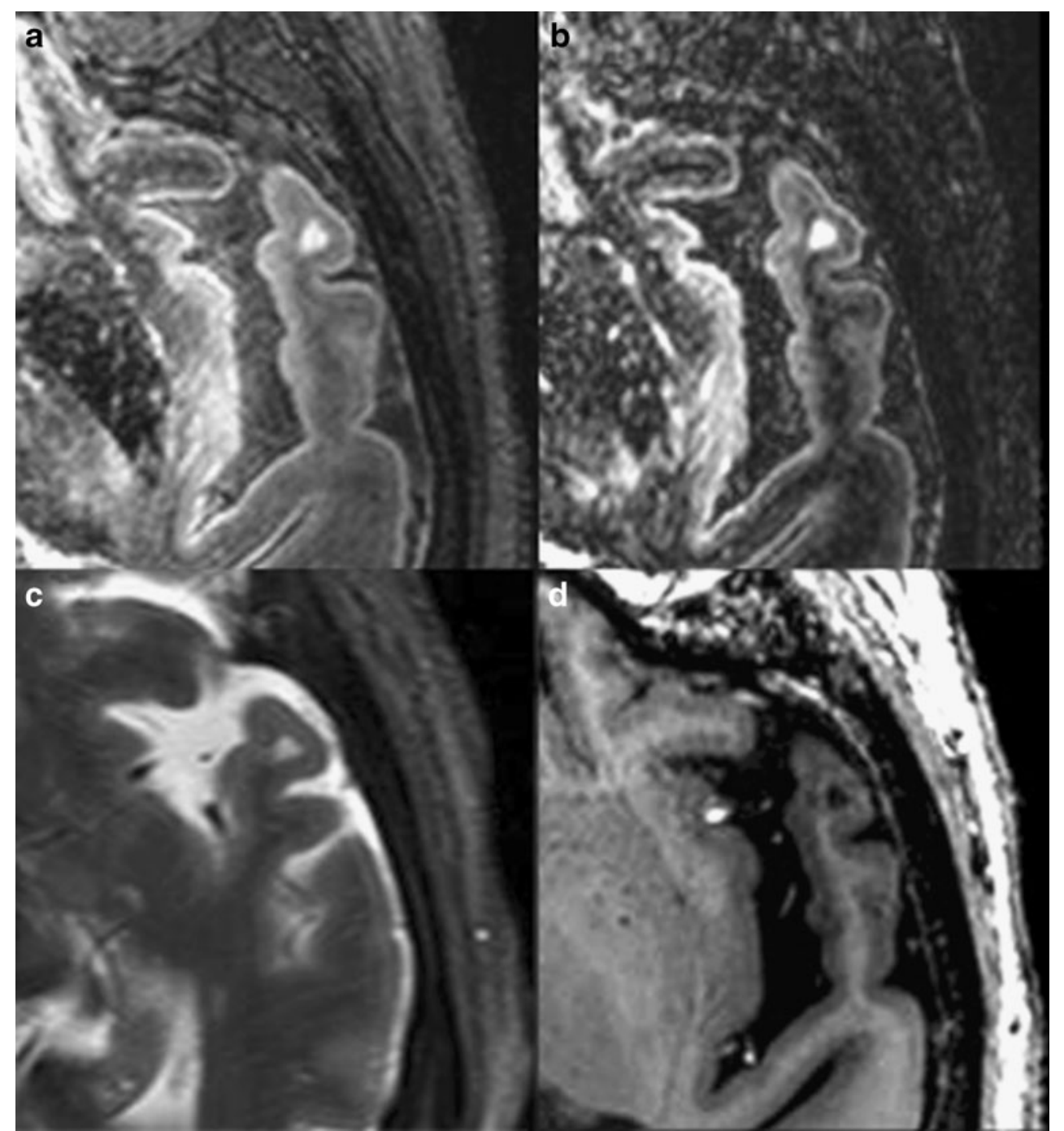

PDT2 by use of a Friedman test and further analysed by a Wilcoxon signed rank test. When significant, results were shown as relative gain compared with 2D-PDT2.

\section{Results}

None of the subjects experienced any additional inconvenience at $7 \mathrm{~T}$ compared with previous examinations they had at $1.5 \mathrm{~T}$ or $3 \mathrm{~T}$.

Evaluation of the acquired images showed incomplete attenuation of CSF in some areas of the brain (Fig. 1) for 3D-MP-FLAIR and 3D-MP-DIR. Perivascular spaces were depicted hyperintense and were not attenuated by the inversion recovery pulses. They were depicted hypointense visible on 3D-T1 images.

The contrast ratio for GM/WM was highest for the 3DMP-DIR (93\%), while the conventional sequences and 3DMP-FLAIR showed intensity differences ranging between
$23 \%$ and $40 \%$ (Table 2). The variation in CR between sequences was much higher for lesions than between GM/ WM. The 3D-MP-DIR and 3D-MP-FLAIR sequences showed in general the highest CR for lesion vs. GM or WM. Only the 2D-T2 sequence had a higher CR for the lesion/WM than either DIR or FLAIR. Lesion contrast (for both GM and WM lesions) was quite low on both 2D-PD and 3D-T1 images, although the negative contrast and sharp delineation on the T1 images helped correct localisation on these images.

Outlined regions of interest (Fig. 2) were drawn in areas with good image quality on the 3D-MP-FLAIR sequence for all subjects and copied to the other sequences. All sequences showed higher signal intensities of frontal and occipital brain areas compared with the temporal lobes for both WM and GM (Fig. 3). However, signal intensities were high enough for proper GM/WM and lesion contrast (Fig. 4). On 3D-MP-FLAIR and 3D-MP-DIR images, the cortex showed an intensity gradient with the outer cortical 
layers being visualized with higher signal intensity compared to the inner layers. (Figure 1a and $\mathrm{b}$ ). In addition to this hyperintense rim, a hypo-intense line was visible in the pre- and post-central cortex, resulting in a banding pattern (Fig. 5). This effect could be seen in all orientations.

\section{Lesion detection}

In each of two healthy controls, both male, 50 and 39 years old, one incidental nonspecific deep WM lesion was found, which was visible on all MR sequences. Lesion counts from MS patients are listed in Table 3. Examples of all lesion types are shown in Fig. 6. Some lesions were only observed on 3D-MP-FLAIR and/or 3D-MP-DIR, but not on 3D-T1 or PD/T2 images. Figure 7 shows an example a lesion that was visible hyperintense on FLAIR and DIR, but not on T1 or PDT2. Overall, the highest number of GM and WM lesions, 592, was detected on the 3D-MP-FLAIR sequence. 3D-MP-DIR showed 558, while 3D-T1- and PDT2-weighted MR images showed 384 and 442 lesions, respectively. Using statistical analysis methods, 3D-MPFLAIR performed better for juxtacortical lesions than PDT2 (205 vs. 125). Both 3D-MP-FLAIR and 3D-MPDIR both performed better than PDT2 for mixed lesions

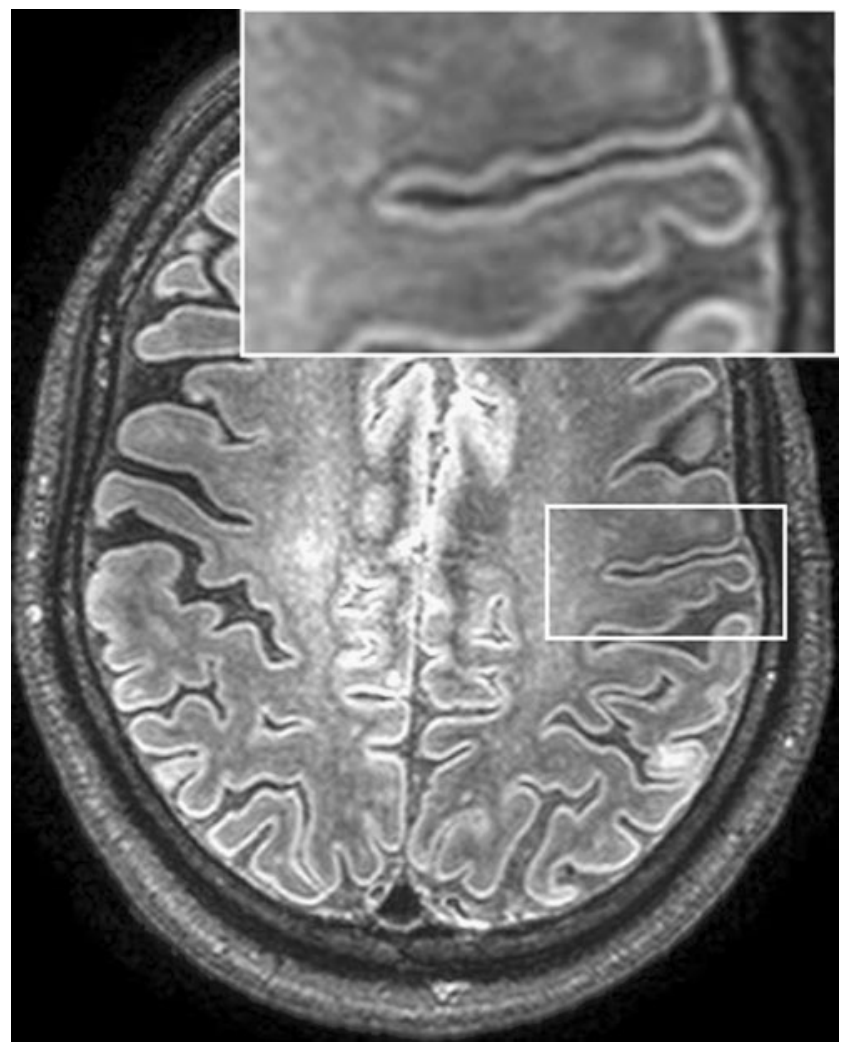

Fig. 5 Axial reformatted 3D-MP-FLAIR image (TR: 8,000 ms, TE: $303 \mathrm{~ms}$, TI: $2,325 \mathrm{~ms}$ ) of a male PP MS patient (50 years of age). Insert shows a magnification of the designated area which shows a banding pattern of the motor cortex as well as a hyper-intense rim
Table 3 Number of lesions observed at $7 \mathrm{~T}$ in $10 \mathrm{MS}$ patients, per sequence and location

\begin{tabular}{lllllll}
\hline & PV & DWM & JC & Mixed & IC & Total \\
\hline 2D-PDT2 & 120 & 155 & 125 & 39 & 3 & 442 \\
3D-T1 & 100 & 131 & 70 & 77 & 6 & 384 \\
3D-MP-FLAIR & 114 & 176 & 205 & 91 & 6 & 592 \\
3D-MP-DIR & 129 & 196 & 133 & 91 & 9 & 558 \\
\hline
\end{tabular}

$P V$ : Lesions in the periventricular area, DWM: Lesions in deep white matter, $J C$ : Lesions in the juxtacortical area, Mixed: Lesions in both white and grey matter, Intra-Cortical: Lesions within the cortex

(both 91 vs. 39). Relative gains of the 3D-MP-FLAIR sequence for the classes with significant difference from the PDT2 sequence were 164\% (juxtacortical) and 233\% (mixed). The relative gain was $233 \%$ for mixed lesions on 3D-MP-DIR. Although not statistically significant, periventricular and deep WM lesions also tended to be best detected on 3D-MP-FLAIR and 3D-MP-DIR images, while 3D-T1 showed the lowest number of WM lesions. Periventricular lesions were well seen on all MR sequences but 3D-MP-DIR showed the highest number (129). Table 4 shows the qualitative scoring of images for motion artefacts and homogeneity. Significant motion artefacts that could have precluded image interpretation (score 5) were not observed.

\section{Discussion}

Using magnetisation preparation, we were able to obtain whole-brain (near-) isotropic, high-resolution 3D-MPFLAIR and 3D-MP-DIR images at $7 \mathrm{~T}$, within clinically feasible MR data acquisition times. These optimised techniques were able to detect higher numbers of MS lesions in both WM and GM, compared with 2D-PDT2 and 3D-T1. Future studies should now compare these new sequences at $7 \mathrm{~T}$ with similar sequences at lower field strength within the same patient population. However, one should notice that pulse sequences that are optimal for lower field strength are not necessarily optimal at high field strength and vice versa. Whereas a $3 \mathrm{~T}$ vs. $7 \mathrm{~T}$ comparison of a 2D-PDT2 only requires slightly different TR and TEs, comparison of 3D-FLAIR and 3D-DIR requires larger modifications such as the prevention of increased, and unwanted, T1-weighting at $7 \mathrm{~T}$ by using magnetization preparation [19, 21].

The RF wavelength at $7 \mathrm{~T}$ is less than half of that at $3 \mathrm{~T}$. Therefore, wavelength becomes a factor that compromises the application of a uniform B1-field, which leads to decreased signal intensity in the temporal lobes when compared with more central brain areas on images of most of the $7 \mathrm{~T}$ sequences. Also a lack of signal intensity can be 


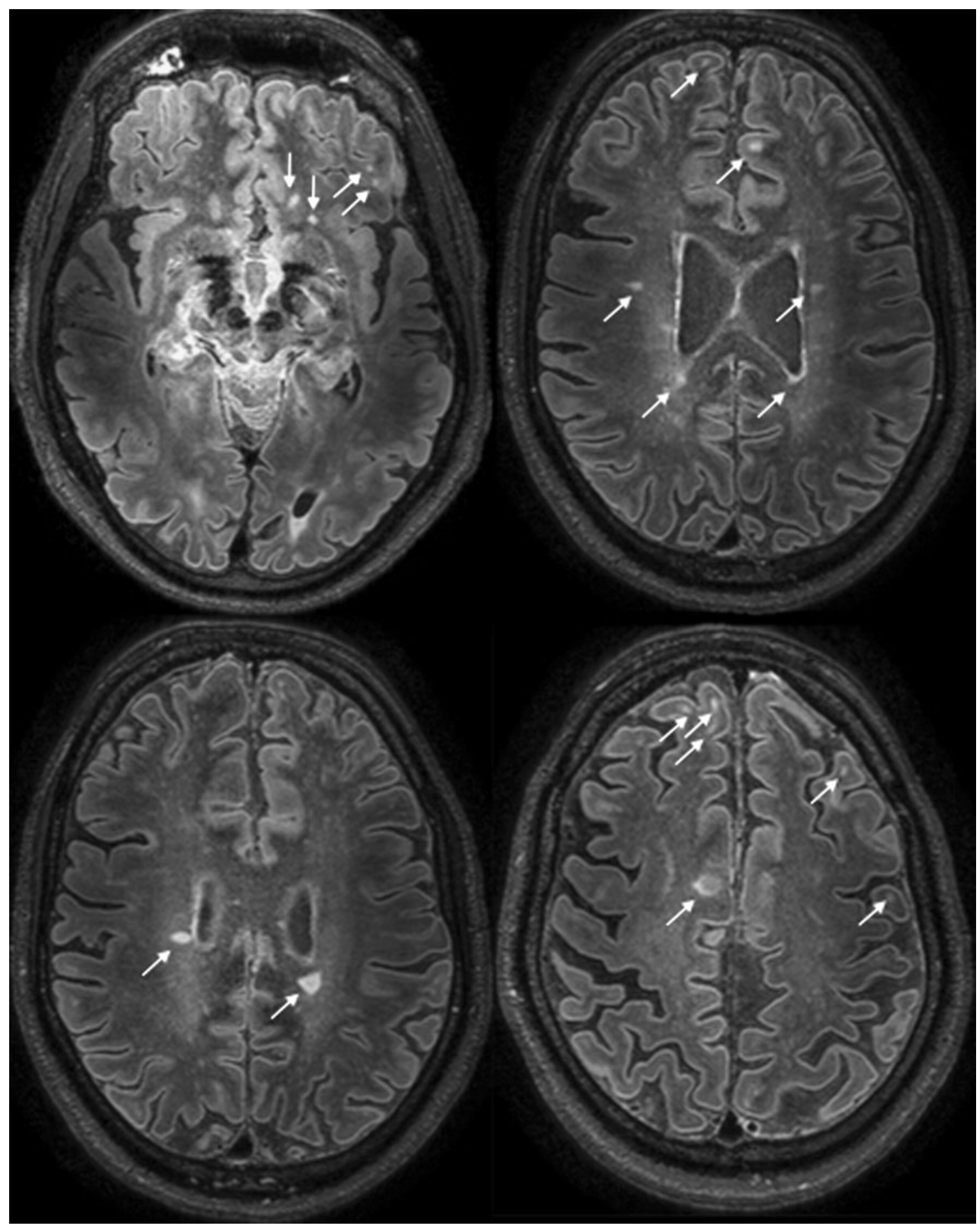

Fig. 6 Axial reformatted 3D-MP-FLAIR images of a 50-year-old male PP MS patient on several levels with a number of lesions indicated by arrows

observed around the fossa posterior. A possible solution for the problem of an heterogeneous B1-field would be the use of multi-transmit/receive coils [22]. Nevertheless, when using the currently available hardware, MS lesions appear with high signal intensity compared with the surrounding tissue even in the temporal lobes. Although lesion detection in the temporal lobes may not be a problem (Fig. 4), the image quality will be enhanced if homogeneous $B_{1}$ would be achieved in the brain by e.g. $B_{1}$ shimming solutions with multiple RF channels. A higher $B_{1}$ in the temporal lobes would lead to higher signal intensities, which would facilitate accurate localization of the lesions. Moreover, the $T_{2}$ weighting of the long TSE train would be stronger (because the refocusing angles would be larger), which would likely 


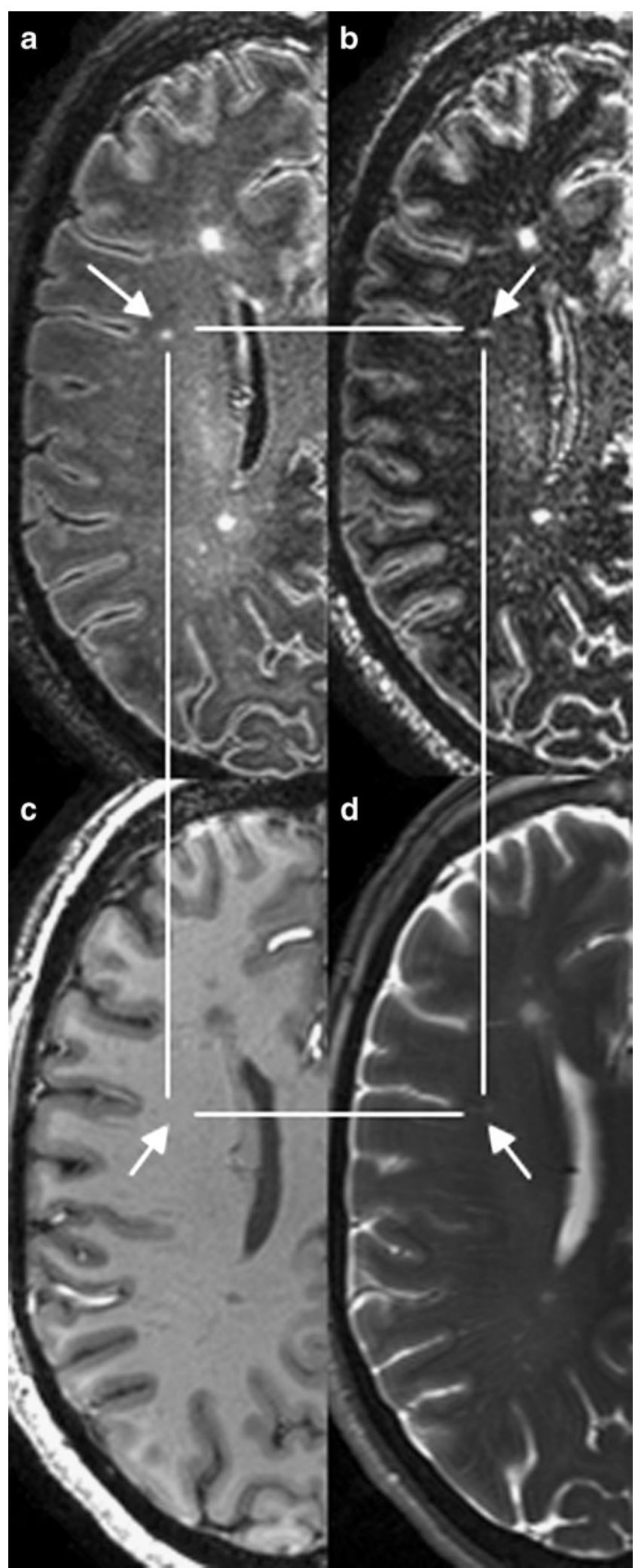

Fig. 7 Appearance of lesions that were visible on 3D-MP-FLAIR and 3D-MP-DIR, but not on PD/T2 or 3D-T1 (a, b and $\mathbf{d}$ reformatted). Top left: 3D-MP-FLAIR, top right: 3D-MP-DIR, bottom left: 2D-T2, bottom right: $3 \mathrm{D}-\mathrm{T} 1$ further increase the lesion conspicuity. When looking at CR values of the FLAIR and DIR sequences, their high values for lesion contrast can be explained by both the inversion pulses and MP: The high CR values for DIR are caused by the suppression of WM. The MP used before the inversion pulses hardly affects CSF or tissue with a high T1 relaxation time. Therefore lesions (with high relaxation times) that are not suppressed by the inversion pulse will neither have lost signal intensity from the MP. Therefore, although the signal intensities of normal GM and WM are lower in the temporal lobes, lesions still have a high conspicuity. Future studies could address the impact of regional contrast effects on observer variability. Despite the use of adiabatic inversion pulses, $B 1$ heterogeneity can cause incomplete spin inversion at certain locations about $80 \%$ inversion at one third of the nominal $B 1$ [19], which may explain the heterogeneity in CSF suppression. As CSF has a very long $T 2$ relaxation time, only a very small amount of remaining longitudinal magnetisation is needed to obtain a considerable but variable signal in the heavily T2-weighted FLAIR and DIR images. Moreover, the higher signal intensity of WM extending from the central sulcus to the thalamus compared with other WM is similar to that observed at lower field strength and is probably due to regional variation of both $\mathrm{T} 1$ and $\mathrm{T} 2$ relaxation times of WM $[23,24]$. These effects were also shown for 7 TT2*-weighted images [25].

All sequences used showed many perivascular spaces at 7 T. For the 2D-PDT2 and 3D-T1 images this may be explained by the high spatial resolution, which reduces partial volume effects. For FLAIR and DIR it may also partly be explained by the fact that fluid in peri-vascular spaces has a different composition compared with CSF, which may shorten T1 relaxation times [26, 27]. Inversion recovery timing would then not meet the $\mathrm{T} 1$ relaxation. The risk of confusing peri-vascular spaces with lesions may occur and therefore it is necessary to follow them over several slices and/or observe them on orthogonal views (the near isotropic resolution of the $3 \mathrm{D}$ acquisition allows reformatting of the data in any desired orientation). Lesions and peri-vasuclar spaces can then be discriminated on their shape: most lesions have an oval, sphere-like appearance, while peri-vascular spaces have an elongated, linear shape and have small diameters over several slices. One of the readers is a board certified neuro-radiologist with over 10 years of experience in neuro-inflammatory diseases and had no problems discriminating lesions from peri-vascular spaces using the described method.

Visualisation of the cortex

To enable correct classification of lesions (especially juxtacortical and mixed lesions), a good GM/WM contrast is necessary. The 3D-MP-FLAIR and 3D-MP-DIR sequences 
Table 4 Image quality scores at $7 \mathrm{~T}$ in MS patients and healthy controls

\begin{tabular}{|c|c|c|c|c|}
\hline Score & $\mathrm{PD} / \mathrm{T} 2$ & 3D-T1 & 3D-MP-FLAIR & 3D-MP-DIR \\
\hline \multicolumn{5}{|l|}{ Patients $(n=10)$} \\
\hline Motion Artefacts [mean (sd)] & $0.6(1.1)$ & $1.5(0.9)$ & $1.4(0.9)$ & $1.5(0.9)$ \\
\hline Homogeneity [mean (sd)] & 2 & $1.7(0.6)$ & $2.1(0.3)$ & $1.6(0.5)$ \\
\hline \multicolumn{5}{|l|}{ Controls $(n=5)$} \\
\hline Motion Artefacts [mean (sd)] & 0 & $1.5(0.6)$ & $0.5(1.0)$ & $0.8(1.0)$ \\
\hline Homogeneity [mean (sd)] & 2 & $1.8(0.5)$ & 2 & 2 \\
\hline
\end{tabular}

Motion Artefacts: 0: none, 1: almost not noticeable, 2: incidental motion, 3: some artefacts, 4: multiple artefacts, needs careful analysis, 5: high numbers of artefacts, prohibits valid analysis

Homogeneity: 0: good homogeneity, 1: almost homogeneous images, 2: only some heterogeneity close to skull base and in temporal lobes, 3 : heterogeneous at multiple locations, 4: high number of heterogeneous areas, 5: highly heterogeneous images, no valid analysis possible

No subjects scored 4 or 5 in any of the categories

showed good contrast between GM and WM, except for some areas, e.g. pre- and post-central gyrus. The hyperintense rim, which represents the outer layers of the cortex, may reduce the visibility of small intra-cortical lesions. Next to the hyper-intense rim also a banding pattern of the cortex is visible, mostly from the motor cortex. This effect has also been observed on DIR images at $3 \mathrm{~T}$ and is expected to visualise layer III and V (both pyramidal) of the motor cortex [28]. The amount of myelin in these layers is higher than in the other layers which may cause the observed effects. The observed contrast within the cortex due to differences in cortical structure has also been shown in several $\mathrm{T} 1$ relaxation time and $\mathrm{T} 2 *$ weighted MRI studies e.g. [14, 23], although more subtly.

\section{Detection of lesions}

Many deep white matter and juxta-cortical lesions in particular were found in MS patients by both 3D-MPFLAIR and 3D-MP-DIR. The number of pure intra-cortical lesions however, was relatively low. This might be caused by the afore-mentioned hyper-intense rim that reduced conspicuity of these lesions, but may also be due to the specific patient group which had a relatively low EDSS score (4.5). To our knowledge no clinical studies so far have been performed using 3D-FLAIR and 3D-DIR at $7 \mathrm{~T}$, possibly because implementation of these sequences is not straightforward at high field strength and needs dedicated modifications of the sequences [19]. A number of lesions were detected on the FLAIR and DIR images but not on the $\mathrm{T} 1$ and $\mathrm{PD} / \mathrm{T} 2$. As for the 2D PD/T2 sequences, this may be caused by the larger slice thickness. For the 3D-T1, with a comparable spatial resolution as the FLAIR and DIR sequences, further study with a one-on-one lesion comparison between sequences may help to explain these differences. The studies published before have used 3D-T1w, 2D-
PDT2 and 2D-T2* ${ }_{\mathrm{W}}$ sequences [29] or worked with ex vivo material [30]. The $\mathrm{T} 2 * \mathrm{~W}$ sequence type did show high conspicuity of lesions, as well as the same WM to GM lesion ratio as observed with histopathology. As the contrast type in $\mathrm{T} 2 *$-weighted images strongly differs from that known from more conventional sequences, it is unknown how the lesion conspicuity on $\mathrm{T} 2 *$ weighted images compares with that in FLAIR and DIR images. A practical complication of $\mathrm{T} 2 *$-weighted images is the long acquisition time that is needed for whole brain coverage. The shorter imaging times in combination with the high contrast of the hyperintense lesions in the imaging types presented in this study may therefore be more feasible for clinical applications in MS.

\section{Study limitations}

Unfortunately, the single transmit coil that was used in this study is unable to induce a fully homogeneous B1 field at 7 T. Possibly the best way to optimise the homogeneity of the images, and improve the degree of CSF suppression, would be to use parallel excitation by use of multiple transmit. If not available, a normalisation filter may be used to obtain more homogeneous images at the cost of some signal intensity and contrast. In this explorative study normalisation was not used so as not to loose any signal intensity. Owing to the presence of the bright rim in the outer layer of the cortex on both 3D-MP-FLAIR and 3DMP-DIR it cannot be excluded that subpial lesions are not being detected. The conspicuity of this cortical lesion type should be validated on post-mortem slices in combination with histopathology. Also a comparison of images of the same type acquired at lower field strength would be valuable to show the additional strength of 7 T MRI in MS.

To conclude, we have shown the feasibility of both 3DMP-FLAIR and 3D-MP-DIR for (cortical) lesion detection at $7 \mathrm{~T}$ with high contrast ratios and high spatial resolution in 
MS patients. Future studies that also look at the relation with clinical impact may further reveal its value in imaging for diagnostics and research.

Acknowledgement Mr. F. Visser is a part-time employee of Philips Healthcare, Best, the Netherlands.

Open Access This article is distributed under the terms of the Creative Commons Attribution Noncommercial License which permits any noncommercial use, distribution, and reproduction in any medium, provided the original author(s) and source are credited.

\section{References}

1. Geurts JJ, Pouwels PJ, Uitdehaag BM et al (2005) Intracortical lesions in multiple sclerosis: improved detection with 3D double inversion-recovery MR imaging. Radiology 236:254260

2. Moraal B, Roosendaal SD, Pouwels PJ et al (2008) Multi-contrast, isotropic, single-slab 3D MR imaging in multiple sclerosis. Eur Radiol 18:2311-2320

3. Wattjes MP, Lutterbey GG, Gieseke J et al (2007) Double inversion recovery brain imaging at $3 \mathrm{~T}$ : diagnostic value in the detection of multiple sclerosis lesions. Am J Neuroradiol 28:54-59

4. Simon B, Schmidt S, Lukas C et al (2010) Improved in vivo detection of cortical lesions in multiple sclerosis using double inversion recovery MR imaging at 3 Tesla. Eur Radiol 20:1675-1683

5. Wattjes MP, Barkhof F (2009) High field MRI in the diagnosis of multiple sclerosis: high field-high yield? Neuroradiology 51:279-292

6. Geurts JJ, Barkhof F (2008) Grey matter pathology in multiple sclerosis. Lancet Neurol 7(841-51):7

7. Rovaris M, Filippi M, Minicucci L et al (2000) Cortical/ subcortical disease burden and cognitive impairment in patients with multiple sclerosis. AJNR Am J Neuroradiol 21:402-408

8. Bagnato F, Butman JA, Gupta S et al (2006) In vivo detection of cortical plaques by MR imaging in patients with multiple sclerosis. AJNR Am J Neuroradiol 27:2161-2167

9. Mcfarland HF (2009) Examination of the role of magnetic resonance imaging in multiple sclerosis: A problem-orientated approach. Ann Indian Neurol 12:254-26310

10. Geurts JJ, Blezer EL, Vrenken H et al (2008) Does high-field MR imaging improve cortical lesion detection in multiple sclerosis? $\mathrm{J}$ Neurol 255(183-91):11

11. Wiesinger F, Van de Moortele P-F, Adriany G et al (2006) Potential and feasibility of parallel MRI at high field. NMR Biomed 19:368-378

12. Wattjes MP, Harzheim M, Lutterbey GG et al (2008) Does high field MRI allow an earlier diagnosis of multiple sclerosis? J Neurol 255(1159-63):13
13. Tallantyre EC, Morgan PS, Dixon JE et al (2009) A comparison of 3T and 7T in the detection of small parenchymal veins within MS lesions. Invest Radiol 44:491-494

14. Hammond KE, Metcalf M, Carvajal L et al (2008) Quantitative in vivo magnetic resonance imaging of multiple sclerosis at 7 Tesla with sensitivity to iron. Ann Neurol 64:707-713

15. Metcalf M, Xu D, Okuda DT et al (2010) High-Resolution phasedarray MRI of the human brain at seven tesla: initial experience in multiple sclerosis patients. J Neuroimaging 20:141-147

16. Mainero C, Benner T, Radding A et al (2009) In vivo imaging of cortical pathology in multiple sclerosis using ultra-high field MRI. Neurology 73:941-948

17. Zwanenburg JJ, Hendrikse J, Visser F et al (2010) Fluid attenuated inversion recovery (FLAIR) MRI at 7.0 Tesla: comparison with 1.5 and 3.0 Tesla. Eur Radiol 20:915-922

18. Madelin G, Oesingmann N, Inglese M (2010) Double inversion recovery MRI with fat suppression at seven Tesla: initial experience. J Neuroimaging 20:87-92

19. Visser F, Zwanenburg JJ, Hoogduin JM et al (2010) Highresolution magnetization-prepared 3D-FLAIR imaging at 7.0 Tesla. Magn Reson Med 64:194-202

20. Polman CH, Reingold SC, Edan G et al (2005) Diagnostic criteria for multiple sclerosis: 2005 revisions to the "McDonald Criteria". Ann Neurol 11:840-846

21. Visser F, Zwanenburg JJ, de Graaf WL et al (2010) 3D Magnetization prepared double inversion recovery (3D MP-DIR) at seven Tesla. Proc ISMRM 2010:2068

22. Fujita H (2007) New horizons in MR technology: RF coil designs and trends. Magnetic resonance in medical sciences. Magn Reson Med Sci 6:29-42

23. Steen RG, Reddick WE, Ogg RJ (2000) More than meets the eye: significant regional heterogeneity in human cortical T1. Magn Reson Imaging 18:361-368

24. van Walderveen MAA, van Schijndel RA, Pouwels PJW et al (2003) Multislice T1 relaxation time measurements in the brain using IR-EPI: reproducibility, normal values, and histogram analysis in patients with multiple sclerosis. JMRI 18:656-664

25. Li T-Q, van Gelderen P, Merkle $\mathrm{H}$ et al (2006) Extensive heterogeneity in white matter intensity in high-resolution T2*-weighted MRI of the human brain at $7.0 \mathrm{~T}$. NeuroImage 32:1032-1040

26. Abbott NJ (2004) Evidence for bulk flow of brain interstitial fluid: significance for physiology and pathology. Neurochem Int 45:545-552

27. Johanson CE, Duncan JA, Klinge PM et al (2008) Multiplicity of cerebrospinal fluid functions: New challenges in health and disease. Cerebrospinal Fluid Res 5:10

28. Kim EY, Kim DH, Chang JH et al (2009) Triple-layer appearance of brodmann area 4 at Thin-section double inversion-recovery MR imaging. Radiology 250:515-522

29. Kollia K, Maderwald S, Putzki N et al (2009) First clinical study on ultra-high-field MR imaging in patients with multiple sclerosis: comparison of $1.5 \mathrm{~T}$ and $7 \mathrm{~T}$. Am J Neuroradiol 30:699-702

30. Pitt D, Boster A, Pei W et al (2010) Imaging cortical lesions in multiple sclerosis with ultra-high-field magnetic resonance imaging. Arch Neurol 67:812-818 